\title{
Optical coherence tomography detection of characteristic retinal nerve fiber layer thinning in nasal hypoplasia of the optic disc
}

\begin{abstract}
Purpose To determine the clinical usefulness of optical coherence tomography (OCT) for detecting thinning of the retinal nerve fiber layer (RNFL) in eyes with nasal hypoplasia of the optic discs (NHOD). Patients and methods The medical records of five patients (eight eyes) with NHOD were reviewed. The ratio of the disc-macula distance to the disc diameter (DM/DD) and the disc ovality ratio of the minimal to maximal DD were assessed using fundus photographs. The RNFL thicknesses of the temporal, superior, nasal, and inferior quadrants were evaluated using OCT quadrant maps.

Results All eight eyes had temporal visual field defects that respected the vertical meridians that needed to be differentiated from those related to chiasmal compression. The mean DM/DD ratio was 3.1 and the mean disc ovality ratio was 0.81 . The mean RNFL thicknesses of the temporal, superior, nasal, and inferior quadrants were 90.3, 103.1, 34.8, and 112.8 microns,

respectively.

Conclusion Small optic discs and tilted discs might be associated with NHOD.

Measurement of the RNFL thickness around the optic disc using OCT scans clearly visualized the characteristic RNFL thinning of the nasal quadrants corresponding to the temporal sector visual field defects in eyes with NHOD. OCT confirmed the presence of NHOD and might differentiate eyes with NHOD from those with chiasmal compression.
\end{abstract}

Eye (2017) 31, 1685-1688; doi:10.1038/eye.2017.134; published online 21 July 2017

M Haruta, R Kodama and R Yamakawa

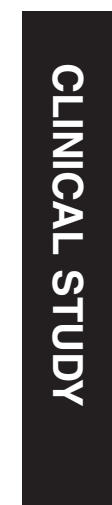

Introduction

Nasal hypoplasia of the optic disc (NHOD) is a congenital anomaly characterized by hypoplasia of the nasal portion of the optic disc with thinning of the retinal nerve fiber layer (RNFL). ${ }^{1-3}$ Visual symptoms include sector visual field defects emerging from the blind spots and extending to the temporal periphery, but patients might not be aware of their visual field defects unless they are tested. NHOD, which can occur either unilaterally or bilaterally, is regarded as a nonprogressive condition.

Although a few case series have been reported, ${ }^{1,2}$ little is known about the clinical features of NHOD.

In the current study, we examined eight eyes of five female patients with NHOD. All eyes had temporal visual field defects that respected the vertical meridians that needed to be differentiated from those related to chiasmal compression. We determined the clinical usefulness of quadrant maps obtained by circumpapillary optical coherence tomography (OCT) scans for detecting the characteristic nasal RNFL thinning in eyes with NHOD.

\section{Subjects and methods}

We reviewed retrospectively the medical records of a total of 43 patients who presented to our neuro-ophthalmology unit with temporal visual field defects from September 2015 to August 2016. The Ethical Committee of Kurume University approved this study. Thirty-four patients with abnormal findings on head magnetic resonance or computed tomographic images were excluded. Four patients with optic
Department of Ophthalmology, Kurume University School of Medicine, Kurume, Fukuoka, Japan

Correspondence: M Haruta, Department of Ophthalmology, Kurume University School of Medicine, 67 Asahi-machi, Kurume, Fukuoka 8300011, Japan

Tel: +81942317574; Fax: +81942370324 E-mail: haruta_masatoshi@ med.kurume-u.ac.jp

Received: 7 January 2017 Accepted in revised form: 16 May 2017 Published online: 21 July 2017 
neuropathies were also excluded. The medical records of five patients (eight eyes) with NHOD were reviewed. All patients underwent complete ocular examinations, including measurement of the best-corrected visual acuity (BCVA) and intraocular pressure (IOP), Goldmann perimetry, slit-lamp biomicroscopy, and dilated fundus examinations. The patients' color fundus photographs and spectral-domain OCT images (RS-3000 Advance OCT, Nidek, Gamagori, Japan) were reviewed. Circumpapillary scans obtained by RS-3000 Advance OCT provided RNFL thickness maps of the temporal, superior, nasal, and inferior quadrants based on the normative database. On the quadrant maps, green indicated that the RNFL thicknesses were within the normal range, yellow abnormal below the 5\% level, and red abnormal below the $1 \%$ level. The ratio of the discmacula distance to the disc diameter (DM/DD) was calculated using fundus photographs as reported previously. ${ }^{4}$ Optic disc tilt was assessed using the disc ovality ratio of the minimal to maximal DD as reported previously. ${ }^{5}$

\section{Results}

Two patients had unilateral involvement, and three were affected bilaterally. Table 1 shows the clinical characteristics including the results of Goldmann perimetry. All patients were female (mean age, 38.8 \pm 10.4 years). The BCVA at the initial visit was 1.2 in six eyes and 1.5 in two eyes. The mean spherical equivalent was $-6.0 \pm 1.7$ diopters; the mean IOP was $14.9 \pm 3.4 \mathrm{~mm} \mathrm{Hg}$. The mean RNFL thicknesses of the temporal, superior, nasal, and inferior quadrants were $90.3 \pm 12.6$, $103.1 \pm 16.6,34.8 \pm 4.1$, and $112.8 \pm 11.2$ microns, respectively. When the RNFL thicknesses were evaluated using the OCT quadrant maps, all eight eyes were coded red (below the 1\% level) in the nasal quadrant, three eyes red and one eye yellow (below 5\% level) in the superior quadrant, and three eyes yellow in the inferior quadrant. The mean DM/DD ratio was $3.1 \pm 0.5$, and the mean disc ovality ratio of the minimal to maximal DD was $0.81 \pm 0.07$. Fundus photographs, OCT images, and Goldmann perimetry results from case 2 are shown in Figure 1.

\section{Discussion}

Hypoplasia of the optic nerve varies in appearance, ranging from nearly total aplasia to subtle segmental hypoplasia. ${ }^{6}$ Patients with segmental optic nerve hypoplasia have normal visual acuities and sector visual field defects corresponding to the hypoplastic areas of the optic disc. ${ }^{7}$ Compared to the superior segmental optic hypoplasia with inferior visual field defects, ${ }^{8}$ NHOD with temporal visual field defects has been reported less frequently. However, the actual prevalence of NHOD might be much higher because patients might be unaware of their visual field defects unless they are tested and because the nasal rim of the optic nerve is difficult to evaluate owing to the emergence of large retinal vessels.

The current OCT scans clearly showed the RNFL thickness around the optic disc and the characteristic RNFL thinning of the nasal quadrants corresponding to the temporal sector visual field defects in eyes with NHOD. When the RNFL thicknesses were evaluated using the OCT quadrant maps, all eight eyes were coded red in the nasal quadrant, whereas none were coded red or yellow in the temporal quadrant. Although RNFL thinning is often difficult to determine during clinical examination using slit-lamp fundus biomicroscopy or red-free fundus photographs, OCT provided useful information for diagnosing patients with NHOD.

Table 1 Clinical characteristics of nasal hypoplasia of the optic disc

\begin{tabular}{|c|c|c|c|c|c|c|c|c|c|c|c|c|c|}
\hline \multirow[t]{2}{*}{ Case } & \multirow{2}{*}{ Eye } & \multirow{2}{*}{$\begin{array}{l}\text { Age } \\
\text { (Years) }\end{array}$} & \multirow{2}{*}{ Gender } & \multirow{2}{*}{$\begin{array}{l}\text { Spherical } \\
\text { equivalent } \\
\text { (D) }\end{array}$} & \multirow{2}{*}{$\begin{array}{c}I O P \\
(m m H g)\end{array}$} & \multirow{2}{*}{$B C V A$} & \multirow{2}{*}{$\begin{array}{l}\text { Visual field } \\
\text { defects }\end{array}$} & \multirow{2}{*}{$\begin{array}{l}D M / \\
D D \\
\text { ratio }\end{array}$} & \multirow{2}{*}{$\begin{array}{l}\text { Disc ovality } \\
\quad \text { ratio }\end{array}$} & \multicolumn{4}{|c|}{ RNFL thickness (microns) } \\
\hline & & & & & & & & & & Temporal & Superior & Nasal & Inferior \\
\hline \multirow[t]{2}{*}{1} & $\mathrm{R}$ & 35 & $\mathrm{~F}$ & -3.5 & 12 & 1.2 & Temporal defect & 2.3 & 0.94 & 78 & 113 & $29^{\mathrm{a}}$ & 115 \\
\hline & $\mathrm{L}$ & & & -2.9 & 15 & 1.2 & Temporal scotoma & 2.5 & 0.86 & 82 & 126 & $36^{\mathrm{a}}$ & $104^{\mathrm{b}}$ \\
\hline \multirow[t]{2}{*}{2} & $\mathrm{R}$ & 25 & $\mathrm{~F}$ & -6.9 & 21 & 1.5 & Temporal depression & 3.1 & 0.69 & 89 & 123 & $41^{\mathrm{a}}$ & 122 \\
\hline & $\mathrm{L}$ & & & -7.4 & 19 & 1.5 & $\begin{array}{c}\text { Temporal defect/ } \\
\text { scotoma }\end{array}$ & 2.8 & 0.80 & 76 & 109 & $32^{\mathrm{a}}$ & 113 \\
\hline \multirow[t]{2}{*}{3} & $\mathrm{R}$ & 38 & $\mathrm{~F}$ & -7.1 & 13 & 1.2 & $\begin{array}{c}\text { Upper temporal } \\
\text { defect }\end{array}$ & 3.8 & 0.80 & 98 & $99^{\mathrm{b}}$ & $40^{\mathrm{a}}$ & $103^{\mathrm{b}}$ \\
\hline & $\mathrm{L}$ & & & -6.6 & 11 & 1.2 & $\begin{array}{l}\text { Lower temporal } \\
\text { defect }\end{array}$ & 3.7 & 0.75 & 107 & $93^{\mathrm{a}}$ & $30^{\mathrm{a}}$ & $99^{\mathrm{b}}$ \\
\hline 4 & $\mathrm{R}$ & 39 & $\mathrm{~F}$ & -7.0 & 12 & 1.2 & Temporal defect & 3.4 & 0.80 & 81 & $86^{\mathrm{a}}$ & $36^{\mathrm{a}}$ & 136 \\
\hline 5 & $\mathrm{~L}$ & 57 & F & -6.8 & 16 & 1.2 & Temporal defect & 3.0 & 0.80 & 111 & $76^{\mathrm{a}}$ & $34^{\mathrm{a}}$ & 110 \\
\hline
\end{tabular}

Abbreviations: BCVA, best-corrected visual acuity; D, diopters; DM/DD ratio, disc-macula distance to disc diameter (DM/DD) ratio; F, female; IOP, intraocular pressure; L, left; R, right; RNFL, retinal nerve fiber layer. ${ }^{\mathrm{a}}$ Abnormal below the $1 \%$ level. ${ }^{\mathrm{b}} \mathrm{Abnormal}$ below the $5 \%$ level. 
a

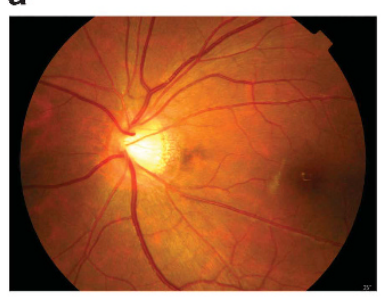

b

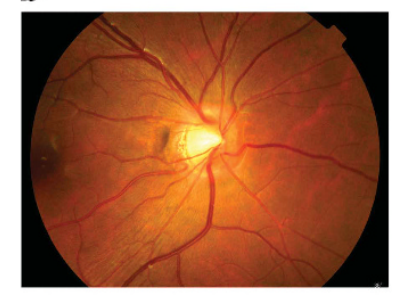

c

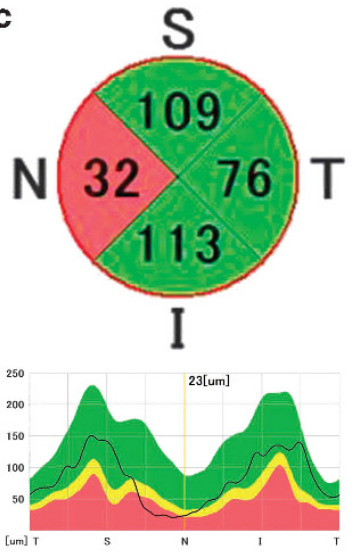

e

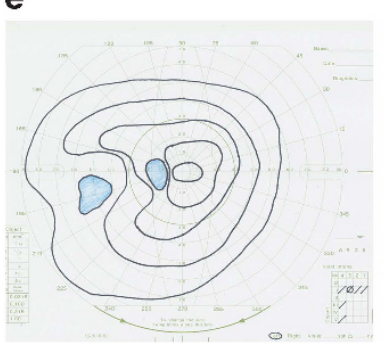

d

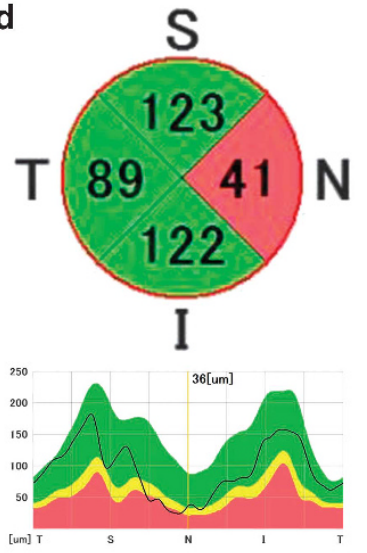

$\mathbf{f}$

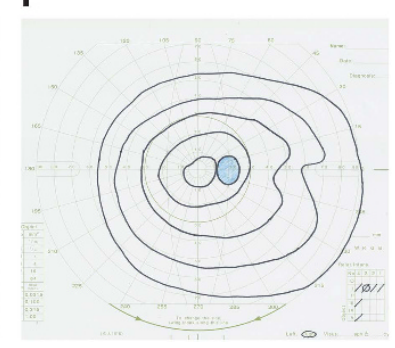

Figure 1 Fundus photographs, optical coherence tomography, and Goldmann perimetry results from case 2. Fundus photographs of the left (a) and right (b) eyes. Quadrant maps and temporal-superior-nasal-inferior-temporal graphs of the thicknesses of the circumpapillary retinal nerve fiber layers in the left (c) and right (d) eyes. Results of Goldmann perimetry of the left (e) and right (f) eyes. T, temporal; S, superior; N, nasal; I, inferior.

Band atrophy of the nasal and temporal disc is a characteristic fundus finding in eyes with bitemporal hemianopia caused by chiasmal compression. Previous studies using OCT quadrant maps in eyes with band atrophy have reported decreased RNFL thickness overall but predominantly in the nasal and temporal quadrants. ${ }^{9}$ However, the current study using OCT quadrant maps in eyes with NHOD suggested that the RNFL thicknesses decreased in the nasal but not in the temporal quadrant. Therefore, careful assessment of the circumpapillary OCT scans might help differentiate eyes with NHOD from those with chiasmal compression, although the visual field defects associated with NHOD might mimic those associated with chiasmal compression.

Segmental optic nerve hypoplasia is thought to be associated with small optic discs and tilted discs. ${ }^{10} \mathrm{~A}$ $\mathrm{DM} / \mathrm{DD}$ ratio of 3.0 or higher has been reported to predict the presence of optic disc hypoplasia in patients with good visual acuities. ${ }^{4}$ In the current study, the mean DM/DD ratio was 3.1, and five of the eight eyes with NHOD had a DM/DD ratio exceeding 3.0. Optic disc ovality has been used as an index to evaluate optic disc tilt, and a disc ovality ratio of 0.8 or lower has been considered significant. ${ }^{5}$ In the current study, the mean disc ovality ratio was 0.81 , and six of the eight eyes with NHOD had a disc ovality ratio of 0.8 or lower. These results suggested that small optic discs and tilted discs also might be associated with NHOD; however, two of the eight study eyes with NHOD had neither a small optic disc nor a tilted disc based on the DM/DD ratio and disc ovality ratio.

Considering the rarity of NHOD, the limitations of the current study included its retrospective nature, the absence of a control group, the small number of cases, and a short follow-up period. We only reviewed the medical records of patients who presented to our neuro-ophthalmology unit with unilateral or bilateral temporal visual field defects, which might have biased the clinical features of NHOD. Nevertheless, we showed that OCT is useful in clinical practice in cases with diagnostic uncertainty to clarify the presence of NHOD and might differentiate eyes with NHOD from those with chiasmal compression. However, larger OCT studies are needed to compare eyes with NHOD with those with chiasmal compression to confirm the current findings.

\section{Summary}

What was known before

- Nasal hypoplasia of the optic disc (NHOD) is a congenital anomaly that is characterized by nasal thinning of the retinal nerve fiber layer (RNFL).

- Visual symptoms include sector visual field defects emerging from the blind spots and extending to the temporal periphery.

What this study adds

- Measurement of RNFL thickness by optical coherence tomography (OCT) scans clearly visualizes the characteristic thinning of the nasal quadrants in eyes with NHOD.

- Careful assessment of OCT scans might differentiate eyes with NHOD from those with chiasmal compression.

\section{Conflict of interest}

The authors declare no conflict of interest. 


\section{References}

1 Buchanan TA, Hoyt WF. Temporal visual field defects associated with nasal hypoplasia of the optic disc. $\mathrm{Br} J$ Ophthalmol 1981; 65: 636-640.

2 Ohguro H, Ohguro I, Tsuruta M, Katai M, Tanaka S. Clinical distinction between nasal optic disc hypoplasia $(\mathrm{NOH})$ and glaucoma with $\mathrm{NOH}$-like temporal visual field defects. Clin Ophthalmol 2010; 4: 547-555.

3 Marsiglia M, Odel JG, Rudich DS, Tsang SH, Plant GT. Photopsia and a temporal visual field defect. Surv Ophthalmol 2016; 61: 363-367.

4 Wakakura M, Alvarez E. A simple clinical method of assessing patients with optic nerve hypoplasia. The disc-macula distance to disc diameter ratio (DM/DD). Acta Ophthalmol (Copenh) 1987; 65: 612-617.

5 Tay E, Seah SK, Chan SP, Lim AT, Chew SJ, Foster PJ et al. Optic disk ovality as an index of tilt and its relationship to myopia and perimetry. Am J Ophthalmol 2005; 139: $247-252$.

6 Lambert SR, Hoyt CS, Narahara MH. Optic nerve hypoplasia. Surv Ophthalmol 1987; 32: 1-9.

7 Petersen RA, Walton DS. Optic nerve hypoplasia with good visual acuity and visual field defects: a study of children of diabetic mothers. Arch Ophthalmol 1977; 95: 254-258.

8 Yamamoto T, Sato M, Iwase A. Superior segmental optic hypoplasia found in Tajimi Eye Health Care Project participants. Jpn J Ophthalmol 2004; 48: 578-583.

9 Kanamori A, Nakamura M, Matsui N, Nagai A, Nakanishi Y, Kusuhara S et al. Optical coherence tomography detects characteristic retinal nerve fiber layer thickness corresponding to band atrophy of the optic discs. Ophthalmology 2004; 111: 2278-2283.

10 Kaur S, Jain S, Sodhi HB, Rastogi A, K. Optic nerve hypoplasia. Oman J Ophthalmol 2013; 6: 77-82. 